\title{
Comparative evaluation of the early effects of the low-level laser therapy versus intra-articular steroids on temporomandibular joint acute osteoarthritis in rats: A histochemical, molecular and imaging evaluation
}

\section{Ocena porównawcza wczesnej reakcji na laser małej mocy w odniesieniu do dostawowego podawania steroidów w ostrym zapaleniu stawów skroniowo- -żuchwowych u szczurów - badanie histochemiczne, molekularne i obrazowe}

\author{
Nermeen AbuBakr ${ }^{A-F}$, Zeinab Salem ${ }^{B-F}$, Zoba Ali ${ }^{A, B, D-F}$, Manal El Assaly ${ }^{A, C-F}$ \\ Department of Oral Biology, Faculty of Dentistry, Cairo University, Egypt \\ A - research concept and design; B - collection and/or assembly of data; $\mathrm{C}$ - data analysis and interpretation; \\ $D$ - writing the article; $E$ - critical revision of the article; $F$ - final approval of the article
}

Address for correspondence

Nermeen AbuBakr

E-mail: nermeen.abubakr@dentistry.cu.edu.eg

Funding sources

None declared

Conflict of interest

None declared

\section{Acknowledgements}

The authors would like to thank Dr. Ahmed Abdalsamad, Assistant Professor at the Department of Oral and Maxillofacial Radiology, Faculty of Dentistry at Cairo University, Egypt, for his help and technical support.

Received on August 8, 2018

Reviewed on September 14,2018

Accepted on September 25, 2018

Published online on December 31, 2018

Cite as

AbuBakr N, Salem Z, Ali Z, El Asaaly M. Comparative evaluation of the early effects of the low-level laser therapy versus intra-articular steroids on temporomandibular joint acute osteoarthritis in rats: A histochemical, molecular and imaging evaluation. Dent Med Probl. 2018;55(4):359-366. doi:10.17219/dmp/96290

DOI

10.17219/dmp/96290

Copyright

○ 2018 by Wroclaw Medical University

and Polish Dental Society

This is an article distributed under the terms of the

Creative Commons Attribution Non-Commercial License

(http://creativecommons.org/licenses/by-nc-nd/4.0/)

\begin{abstract}
Background. Osteoarthritis $(\mathrm{OA})$ is a serious degenerative joint disease. It is one of the main causes of disability in the world. Current treatment modalities have numerous side effects. Subsequently, health experts are looking for alternative therapies.

Objectives. The aim of the study was to evaluate the early effects of low-level laser therapy (LLLT) vs intra-articular (IA) corticosteroids (CS) on acute temporomandibular joint osteoarthritis (TMJOA).

Material and methods. Sixty rats were divided into 3 groups: group 1- untreated OA; group 2 - OA treated with CS; and group $3-O A$ treated with LLL. Half of the animals in each group were sacrificed at 1 and 4 weeks post treatment. The temporomandibular joint was dissected and evaluated histochemically, using quantitative real-time polymerase chain reaction (qRT-PCR), and radiographically.

Results. Histochemically, Safranin-0 staining revealed an obvious reduction in proteoglycans in the untreated osteoarthritic group. However, both of the treated groups showed a moderate increase in glycosaminoglycan (GAG) staining. As for the gRT-PCR results, caspase-3 showed the highest mean value in the untreated $\mathrm{OA}$ group, followed by the CS group, while the lowest mean value was recorded in the LLL group. Radiographically, the condyle showed erosion, flattening, osteophyte formation, and sclerosis in the untreated group, but there was great improvement in both of the treated groups.

Conclusions. Both laser and cortisone showed reparative and formative effects, as evidenced by the increases in the proteoglycan content. However, LLL was superior in its anti-apoptotic effects. Cone beam computed tomography (CBCT) is a valuable tool in assessing osseous abnormalities.
\end{abstract}

Key words: osteoarthritis, temporomandibular joint, glucocorticoids, low-level laser therapy, caspase-3

Słowa kluczowe: zapalenie stawów, staw skroniowo-żuchwowy, glikokortykosteroidy, terapia laserem małej mocy, kaspaza-3 


\section{Introduction}

Osteoarthritis (OA) is a degenerative disease that commonly affects the temporomandibular joint (TMJ). It is identified by the disintegration of the articular cartilage and the underlying subchondral bone. Progressive disintegration of TMJ limits basic daily activities, such as talking and chewing. It also causes discomfort, joint pain, limitation in mandibular movements, and stiffness. This eventually leads to the deterioration of the patient quality of life. ${ }^{1}$

Degradation in cartilage collagens and proteoglycans leads to fibrillation and fissures in the superficial layer of the cartilage. This spreads to deeper layers and finally expands to form erosion. ${ }^{2}$ Apoptotic cell death of chondrocytes has been detected in the osteoarthritic cartilage. This is associated with matrix degeneration and calcification. ${ }^{3}$

Temporomandibular joint osteoarthritis (TMJOA) can be diagnosed both clinically and radiographically. The clinical symptoms are mainly tenderness and pain. The radiographic signs include cortical bone erosion, flattening of joint compartments and bony changes, such as sclerosis and osteophyte formation. ${ }^{4}$

It was proven in the 1940 s that corticosteroids (CS) had strong anti-inflammatory properties. Both natural and synthetic steroids have become the most commonly used anti-inflammatory medications worldwide. ${ }^{5}$ However, repeated intra-articular (IA) CS injections cause pain, blushing of the skin, swelling at the injection site, and an increased risk of infection. ${ }^{6}$ The available forms of IA drug delivery usually necessitate repeated injections, which generates high costs and affects the patient quality of life. ${ }^{7}$

Due to its non-invasiveness and the advantage of provoking nearly no adverse effects, low-level laser therapy (LLLT) has been broadly used to alleviate pain in many musculoskeletal disorders. ${ }^{8}$ It is considered a promising therapeutic intervention, mostly because of its stimulatory effects on tissue metabolism and the ability to regulate the inflammatory process after injury. Other effects include better cellular oxygenation, the release of neurotransmitters associated with pain modulation, and the release of anti-inflammatory and endogenous mediators. ${ }^{9}$

The PubMed and Google Scholar search results from 2008 to 2018 showed that the assessments of the therapeutic effects of LLLT on OA in previous clinical studies were focused mainly on monitoring the improvement in mandibular movements and pain reduction. Previous studies assessed the late effects of LLLT and CS on chronic joint disorders. No radiographic studies have been performed to assess the improvement in bony structures after LLLT. Previous comparisons were made between laser and systemic CS.

The aim of the study was to use an experimental animal model to accurately estimate the early effects of LLLT vs local injections of CS on acute TMJOA. This was assessed histochemically, on a molecular basis and radiographically.

\section{Material and methods}

\section{Ethical statement}

All the experiments were conducted in the animal house at the Faculty of Medicine at Cairo University, Egypt, in accordance with the recommendations and with the approval of the Ethics Committee on Animal Experimentation of the Faculty of Dentistry at Cairo University (approval No.16/9/15).

\section{Animals}

Sixty healthy adult female albino rats, approx. 3-6 months old and weighing about 180-200 grams were used in this study. The animals were housed in a sterile, controlled environment (temperature $23 \pm 5^{\circ} \mathrm{C}$; 12-hour dark/light cycles). They were fed with a standard pellet diet and tap water ad libitum. They were kept individually in stainless steel cages. The animals were randomly distributed into 3 groups of 20 animals each, as shown in Table 1.

\section{Induction of osteoarthritis}

To induce $\mathrm{OA}$ in the TMJ of the rats, a single IA injection of $2 \mathrm{mg}$ /joint of monosodium iodoacetate - MIA (Sigma-Aldrich Chemical Co., St. Louis, USA) dissolved in $50 \mu \mathrm{L}$ sterile $0.9 \%$ saline was administered into the right TMJ of each rat. ${ }^{10}$

\section{Confirmation of osteoarthritis induction}

After 7 days, blood samples were collected once from all the animals. We used the orbital sinus blood sample collection method as one of the general commonly accepted methods of blood collection, approved by the Ethics Com-

Table 1. Experimental groups

\begin{tabular}{|l|c|cc}
\multicolumn{1}{|c|}{ Study design } & Group 1 (OA) & Group 2 (CS) & Group 3 (LLL) \\
\hline Number of rats & 20 & 20 & 20 \\
OA induction & & IA injection of 2 mg of MIA & LLLT \\
Treatment & no treatment & IA injection of dexamethasone & 1 and 4 weeks after LLLT \\
\hline Date of sacrifice & 1 and 4 weeks after OA confirmation & 1 and 4 weeks after dexamethasone administration
\end{tabular}

CS - corticosteroid; IA - intra-articular; LLL - low-level laser; LLLT - low-level laser therapy; MIA - monosodium iodoacetate; OA - osteoarthritis. 
mittee on Animal Experimentation. It yields a moderate to large volume of blood. It is rapid and sterile samples can be obtained. It was performed by inserting a heparinized capillary tube gently but firmly at the medial canthus of the eye into the retro-orbital plexus for the biochemical estimation of C-reactive protein (CRP). The normal CRP value reported in previous studies was $300-600 \mu \mathrm{g} / \mathrm{mL}$. The animals presenting CRP levels above normal were included in our study. ${ }^{11}$ In our experiment, the mean CRP value \pm standard deviation (SD) in the rats was $722.8 \pm 0.57 \mu \mathrm{g} / \mathrm{mL}$.

\section{Dexamethasone administration}

Seven days after the induction of OA, the animals in group 2 were sedated and given a single IA injection of $1.2 \mathrm{mg} / \mathrm{kg}$ dexamethasone (Amriya Pharmaceutical Industries, Alexandria, Egypt). ${ }^{12}$

\section{Laser application}

For laser irradiation, an $\mathrm{Epic}^{\mathrm{Tm}} 10$ indium gallium arsenide phosphide (InGaAsP) system laser (Biolase, Irvine, USA) was used. The rats in group 3 received mild chloroform sedation and the tissue over the TMJ region, 5-10 $\mathrm{mm}$ posterior to the lateral eye canthus, was irradiated. Seven sessions of $60 \mathrm{~s}$ of low-level laser (LLL) were performed every other day during a 2-week period. The physical parameters shown in Table 2 were selected in accordance with a study performed by Khozeimeh et al. ${ }^{13}$ The laser was applied at 3 points: the capsule, the retrodiscal area and the condylar neck. ${ }^{14}$

\section{Animal sacrifice and tissue preparation}

The animals were sacrificed by intraperitoneal injections of $500 \mathrm{mg} / \mathrm{kg}$ sodium pentobarbital, as approved by the Ethics Committee on Animal Experimentation. ${ }^{15}$ The temporomandibular joint was then dissected. The tissues were preserved in $10 \%$ neutral buffered formalin for $24 \mathrm{~h}$, then dehydrated in ascending grades of ethyl alcohol, immersed in xylene, and embedded in paraffin wax. Sections about 3-4-micrometer-thick were prepared.

Table 2. The parameters of the laser device

\begin{tabular}{|lc|}
\multicolumn{1}{|c|}{ Characteristics } & Explanation \\
\hline Laser type & InGaAsP diode laser \\
Brand & Biolase \\
Model number & Epic 10 \\
Wavelength & $940 \mathrm{~nm}$ \\
Operation mode & continuous wave \\
Power output & $0.2 \mathrm{~W}$ \\
Spot size & $300 \mu \mathrm{m}$ \\
Energy & $4 \mathrm{~J}$ \\
Irradiation time & $20 \mathrm{~s} / \mathrm{point}$ \\
\hline
\end{tabular}

InGaAsP - indium gallium arsenide phosphide.

\section{Investigation techniques}

\section{Histochemical examination (Safranin-O staining)}

Safranin-O, used in the articular cartilage staining, binds to glycosaminoglycans (GAGs) and shows an orange-red color. The intensity of the staining demonstrates the proteoglycan content in the cartilage tissue - a deep red color represents a normal cartilage, while orange and faint red colors represent a loss of proteoglycans.

\section{Quantitative real-time polymerase chain reaction for caspase-3 expression}

The mRNA expression level was quantified by quantitative real-time polymerase chain reaction (qRT-PCR). From each sample 1000 ng of the total RNA was used for complementary DNA (cDNA) synthesis by reverse transcription, using a high-capacity cDNA reverse transcriptase kit (Applied Biosystems, Foster City, USA). Complementary DNA was subsequently amplified with a Syber Green I PCR Master Kit (Fermentas, Waltham, USA) in a 48-well plate, using a Step One instrument (Applied Biosystems) as follows: $10 \mathrm{~min}$ at $95^{\circ} \mathrm{C}$ for the enzyme activation, followed by 40 cycles of $15 \mathrm{~s}$ at $95^{\circ} \mathrm{C}, 20 \mathrm{~s}$ at $55^{\circ} \mathrm{C}$ and $30 \mathrm{~s}$ at $72^{\circ} \mathrm{C}$ for the amplification step. Changes in the expression of each target gene were normalized in regard to the mean critical threshold (CT) values of the glyceraldehyde-3-phosphate dehydrogenase - GAPDH housekeeping gene, using the $\Delta \Delta \mathrm{Ct}$ method. We used $1 \mu \mathrm{M}$ of both of the primers specific for each target gene. The primer sequences and annealing temperatures specific for each gene are presented in Table 3.

\section{Cone beam computed tomography examination}

The head of a rat, stored in a plastic container filled with formalin, was placed on the ProMax ${ }^{\circledR} 3 \mathrm{D}$ Mid CBCT machine (Planmeca Oy, Helsinki, Finland), located at the Department of Oral and Maxillofacial Radiology, Faculty of Dentistry at Cairo University, with the use of laser guiding beams. The rat's head was imaged using the following parameters: $90 \mathrm{kVp}, 10 \mathrm{~mA}$, field of view (FOV) of $4 \mathrm{~cm} \times 5 \mathrm{~cm}$, and resolution of a voxel size of $100 \mu \mathrm{m}$, at an exposure time of $12 \mathrm{~s}$. The CBCT images were

Table 3. Primer sequences and annealing temperatures specific for each gene

\begin{tabular}{|l|c|c|} 
Target gene & Primer sequence: 5'-3' $^{\prime}$ & $\begin{array}{c}\text { Gene bank } \\
\text { accession } \\
\text { number }\end{array}$ \\
\hline Caspase-3 & $\begin{array}{c}\text { forward:TGACAGCCAGTGAGACTTGG } \\
\text { reverse: GACTCTAGACGGCATCCAGC }\end{array}$ & NM004346.3 \\
GAPDH & $\begin{array}{c}\text { forward:CACCCTGTTGCTGAGCCATATTC } \\
\text { reverse: GACATCAAGAAGGTGTGAGCAG }\end{array}$ & XR598347.1 \\
\hline
\end{tabular}

GAPDH - glyceraldehyde-3-phosphate dehydrogenase. 
acquired in the Digital Imaging and Communications in Medicine (DICOM) format and assessed using a Romexis Viewer v. 4.6.2.R (Planmeca Oy). On the axial image, the long axis of the sagittal image was aligned to pass along the long axis of the condyle in an anteroposterior direction, so the sagittal image became a true sectional image aligned with the direction of the condyle. Sagittal images of 1-millimeter thickness were used to assess various bony changes affecting the condyle (Fig. 1).

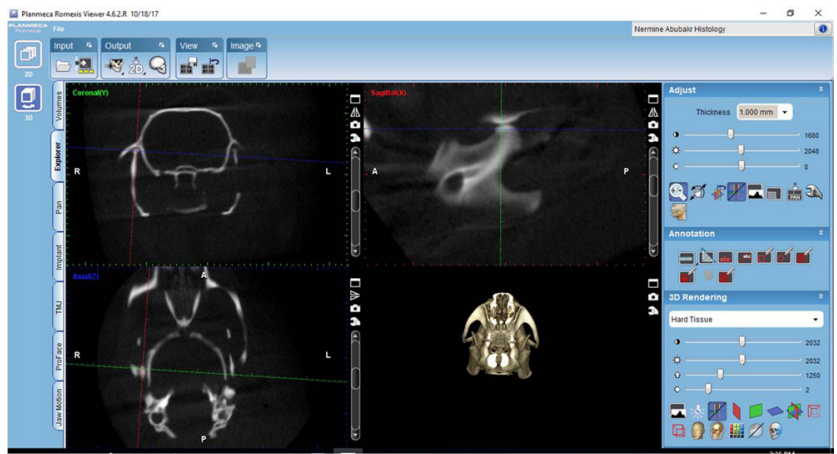

Fig. 1. A photomicrograph showing the method by which the condyle was imaged using cone beam computed tomography (CBCT)

\section{Statistical analysis}

Values were presented as mean and SD. Data was checked for normality, using the Kolmogorov-Smirnov test of normality. The results indicated that most of the data was normally distributed (parametric data); therefore, the one-way analysis of variance (ANOVA) test was used to compare between the groups. This was followed by Tukey's post hoc test when the difference was found to be significant. The unpaired $t$-test was used to compare the same treatment group on the 2 observation dates. The significance level was set at $p<0.05$. The statistical analysis was performed using the SPSS v. 16.0 software for Windows (IBM Corp., Armonk, USA).

\section{Results}

\section{Histochemical results (Safranin-O staining)}

\section{Group 1 (untreated osteoarthritis)}

At 1 week: Safranin-O staining showed a moderate reduction in GAG staining, presented as a faint red color, distributed along the fibrocartilage layer of the temporal bone, disc and condyle. Some areas of a generalized loss in GAG staining, presented as an orange color, were observed in the cartilage layer of the condyle (Fig. 2A).

At 4 weeks: Safranin-O staining showed an obvious reduction and a generalized loss in proteoglycan staining, presented as an orange color and distributed along the fibrocartilage layer of the temporal bone, disc and condyle (Fig. 2B).

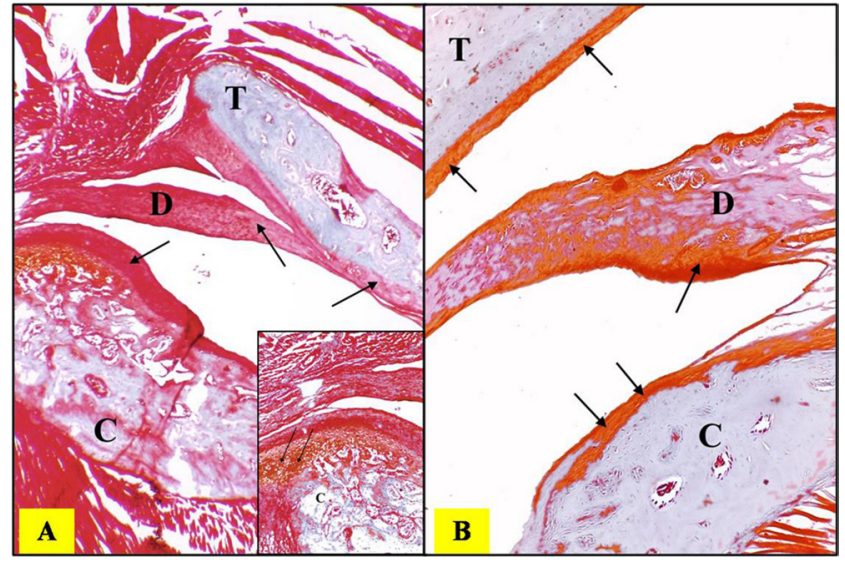

Fig. 2. A photomicrograph of the sagittal section of the temporomandibular joint (TMJ) from the untreated osteoarthritis (OA) group: A - 1 week, showing a moderate reduction in glycosaminoglycan (GAG) staining, presented as a faint red color (arrows) (Safranin-O, $\times 40$ ); inset - an obvious reduction in GAG staining, seen as an orange color (arrows) (Safranin-O, $\times 100$ ); $B-4$ weeks, showing an obvious reduction in GAG staining, presented as an orange color (arrows) (Safranin-O, $\times 100$ )

$\mathrm{C}$ - the condyle; $\mathrm{D}$ - the disc; $\mathrm{T}$ - the temporal bone.

\section{Group 2 (intra-articular corticosteroids)}

At 1 week: Safranin-O staining of the cartilaginous extracellular matrix appeared fairly homogenous, with moderate GAG staining, presented as a red color along the fibrocartilage layer of the temporal bone, disc and condyle (Fig. 3A).

At 4 weeks: Safranin-O staining showed a moderate increase in GAG staining, appearing as a red color in the fibrocartilage layer of the temporal bone, disc and condyle (Fig. 3B).

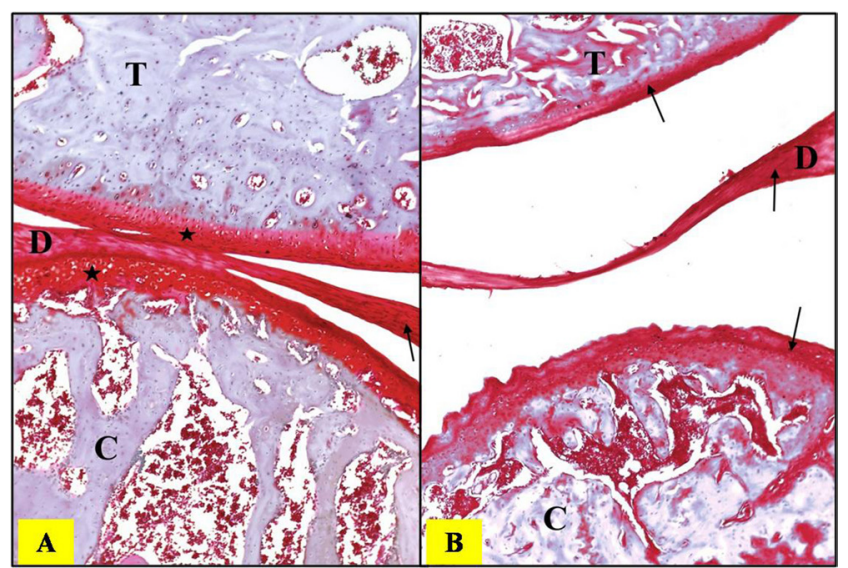

Fig. 3. A photomicrograph of the sagittal section of TMJ from the corticosteroid (CS) group: A - 1 week, showing moderate and homogenous staining, presented as a red color (asterisks and arrow); B - 4 weeks, showing a moderate increase in GAG staining, presented as a red color (arrows) (Safranin-O, $\times 100$ )

\section{Group 3 (low-level laser therapy)}

At 1 week: Safranin-O staining showed a uniform moderate increase in GAG staining, presented as a red color seen in the fibrocartilage layer of the temporal bone, disc 
and condyle. Areas of weak staining, appearing as an orange color, were observed in the condylar head, just beneath the fibrous covering (Fig. 4A).

At 4 weeks: Safranin-O staining of the cartilaginous GAG was intense and homogenous, presenting as a deep red color along the fibrocartilage layer of the temporal bone, disc and condyle, indicating intact aggrecan content (Fig. 4B).

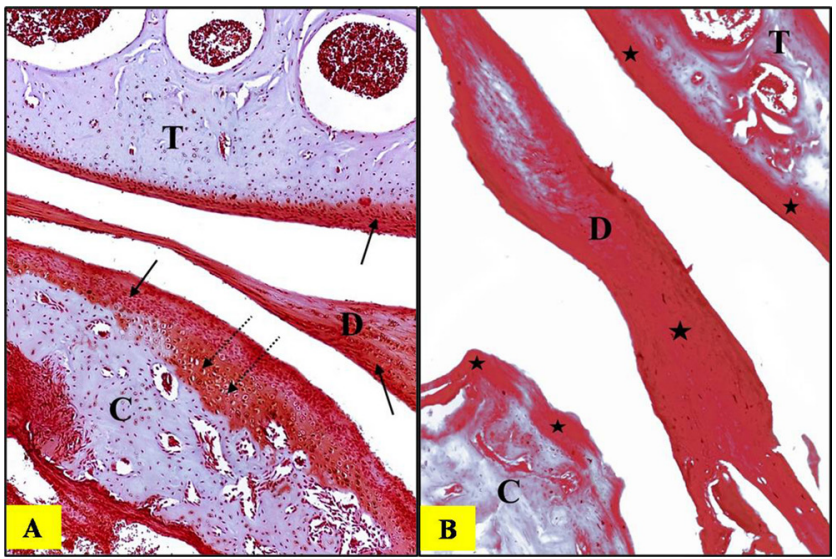

Fig. 4. A photomicrograph of the sagittal section of TMJ from the low-level laser (LLL) group: A - 1 week, showing a moderate increase in GAG staining, presented as a red color (arrows); areas of weak staining appearing as an orange color (dotted arrows) were observed; B - 4 weeks, showing an intense increase in GAG staining, presented as a deep red color (asterisks) (Safranin-O, $\times 100$ )

\section{Quantitative real-time polymerase chain reaction results (caspase-3)}

At both 1 and 4 weeks, the highest mean value was recorded in the untreated OA group, followed by the CS group; the lowest value was recorded in the LLL group. The ANOVA test revealed that the differences between the 3 groups were highly statistically significant $(p=0.001)$ at week 1 ; at week 4 , the differences were extremely statistically significant $(p<0.0001)$. Tukey's post hoc test revealed no significant difference between the CS and LLL groups (Tables 4,5).

\section{Cone beam computed tomography examination results}

The sagittal images obtained by $\mathrm{CBCT}$ revealed erosion, flattening in the articular surface of the condylar head and osteophyte formation in the untreated OA group at week 1 (Fig. 5A). At week 4, sclerosis was observed in the condylar head (Fig. 5B). In the CS group, at week 1, erosion was observed in the condylar head (Fig. 5C); at week 4, minimal sclerosis was observed (Fig. 5D). As for the LLL group, at week 1, minimal erosion was observed in the condylar head (Fig. 5E); at week 4, minimal sclerosis was observed (Fig. 5F).
Table 4. Caspase-3 relative quantitation at 1 week (ANOVA test)

\begin{tabular}{|cc|c|c|}
\multicolumn{1}{c}{ Groups } & & Mean & SD \\
\hline \multirow{2}{*}{1 week } & OA1 & $3.37^{\mathrm{a}}$ & 0.17 \\
& CS1 & $2.71^{\mathrm{b}}$ & 0.45 \\
F-value & LLL1 & $2.37^{\mathrm{b}}$ & 0.17 \\
p-value & & 14.573 & \\
\hline
\end{tabular}

SD - standard deviation; * statistical significance $(p<0.05)$; Tukey's post hoc test - means sharing the same superscript letter are not significantly different.

Table 5. Caspase-3 relative quantitation at 4 weeks (ANOVA test)

\begin{tabular}{|c|c|c|c|}
\hline \multicolumn{2}{|c|}{ Groups } & Mean & $\mathrm{SD}$ \\
\hline \multirow{3}{*}{4 weeks } & OA4 & $3.63^{a}$ & 0.24 \\
\hline & CS4 & $2.64^{b}$ & 0.35 \\
\hline & LLL4 & $2.27^{b}$ & 0.13 \\
\hline F-value & \multicolumn{3}{|c|}{37.948} \\
\hline$p$-value & \multicolumn{3}{|c|}{$<0.0001^{*}$} \\
\hline
\end{tabular}

* statistical significance ( $p<0.05)$; Tukey's post hoc test - means sharing the same superscript letter are not significantly different.
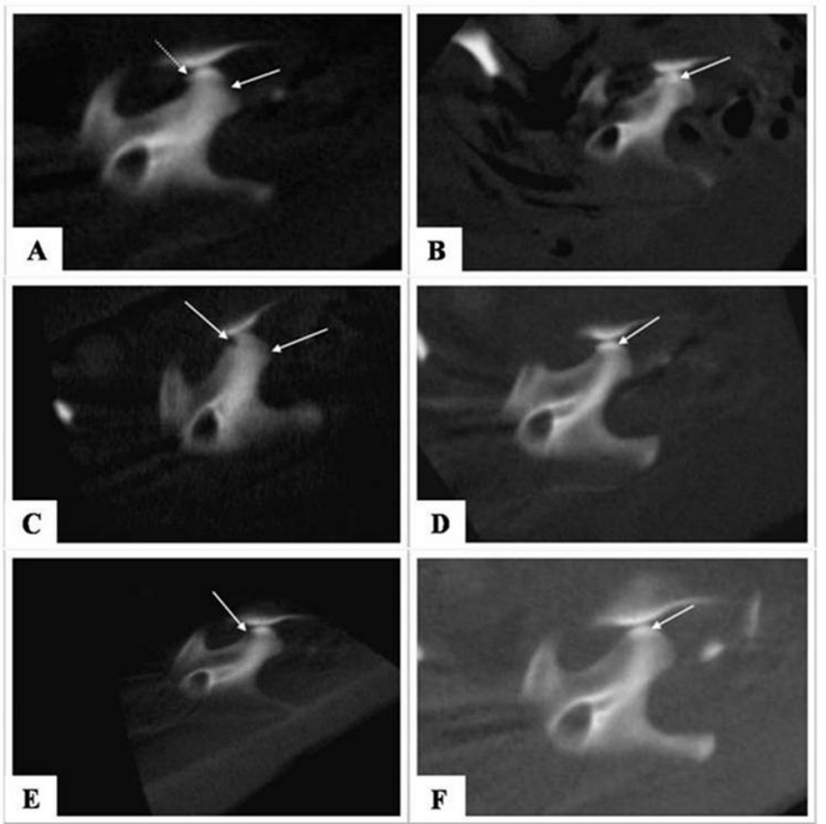

Fig. 5. The sagittal section of the condyle: A - the untreated OA group, 1 week, showing erosion and flattening in the condylar head (arrow) and osteophytes (dotted arrow); B - the untreated OA group, 4 weeks, showing sclerosis in the condylar head (arrow); C: the CS group, 1 week, showing erosion in the condylar head (arrows); D - the CS group, 4 weeks, showing minimal sclerosis (arrow); $\mathrm{E}$ - the LLL group, 1 week, showing minimal erosion in the condylar head (arrow); F - the LLL group, 4 weeks, showing minimal sclerosis in the condylar head (arrow)

\section{Discussion}

Due to the expanding predominance of OA and the absence of cure, it is becoming an increasing burden for countries worldwide. Therefore, enhancements in the prevention and treatment of the disease are urgently 
needed. Human clinical studies have many limitations. The unexpected and slow course of the disease and the late appearance of clinical symptoms in human patients prevents precise investigation of the structural changes and molecular events within the joint. In our study, we used an in vivo preclinical animal model to accurately evaluate the early effects of LLLT vs CS on acute TMJOA - histochemically, on a molecular basis and radiographically. Without the use of animal models, the limitations inherent to clinical trials would hinder the ongoing progress in learning about and treating the disease. ${ }^{16}$

In the current study, we used MIA to induce OA. The IA injection of MIA resulted in the disorganization of the articular cartilage by hindering the activity of GAPDH in chondrocytes. This eventually led to the disruption of glycolysis and cell death. These chondrocytes have nearly identical histopathology as compared to human OA. The MIA-induced OA animal model almost exactly mimics the pain and structural changes accompanying the disease in human patients. ${ }^{17}$

We started treatment 7 days after the confirmation of OA. Guzman et al. observed extensive chondrocyte degeneration and the collapse of the cartilaginous matrix with a marked loss of chondrocyte by 5 or 7 days. ${ }^{17}$

The confirmation of OA induction in our study was performed through a biochemical analysis of CRP. Several studies have proven the presence of a relationship between CRP and OA, in which the elevated values of acute phase proteins, especially CRP, were correlated with the disease. C-reactive protein is widely accepted as a clinical measure of disease activity. Its use as a marker of arthritic disease has been supported in many studies. Connolly et al. reported that the CRP values were significantly higher in arthritic rats than in normal controls, ${ }^{11}$ which concurred with the results obtained in our study.

In our experiment, dexamethasone was used as a standard, traditional way to treat OA. Corticosteroids are gold standard anti-inflammatory agents. Their effects are mediated through binding to the glucocorticoid receptor, which translocates from the cytoplasm to the nucleus and binds to glucocorticoid response elements. This regulates the transcription of certain genes, thus reducing pro-inflammatory cytokine synthesis. ${ }^{18}$

Low-level laser therapy is a treatment modality that has been used as alternative to the traditional CS treatment. It has an extensive variety of effects at the molecular, cellular and tissular levels. Within the cell, LLLT increases the adenosine triphosphate (ATP) production in the mitochondria, modulates reactive oxygen species (ROS) and induces transcription factors. These transcription factors synthesize proteins that trigger more downstream effects, such as increased cell proliferation, migration and tissue oxygenation, and the modulation of the levels of cytokines, growth factors and inflammatory mediators. ${ }^{19}$

The articular cartilage is made of chondrocytes and extracellular matrix, consisting mainly of proline, hydroxyproline, phenylalanine, GAGs, and proteoglycans. Using
Safranin-O, a detailed cartilage histology is shown. The concentration and intensity of the red staining of the articular cartilage measures the GAG content. ${ }^{20}$

Our histochemical results coincide with those of Wang et al., who observed cartilage degeneration following the IA injection of MIA, detectable as a loss of Safranin-O staining, indicating a reduction in GAGs in the cartilage and a reduction in chondrocytes. ${ }^{10}$

Our findings are also in agreement with a study by Lu et al., in which the CS treatment had a protective effect on the articular cartilage in models of OA. ${ }^{21}$

Gottlieb et al. evaluated the influence of LLLT on the proteoglycan content in the arthritically changed cartilage in animals and found that the changes in the irradiated joints were less severe, and that LLLT accelerated the metabolic rate in the hyaline cartilage and increased proteoglycan synthesis. ${ }^{22}$ This was compatible with the findings of our study.

Regarding the qRT- PCR results, the apoptotic marker caspase-3 showed the highest value in the untreated osteoarthritic group. This is consistent with a study of discectomy-induced TMJOA conducted by Kourí-Flores et al., who demonstrated that gene expression of the death receptor family and immuno-histochemical staining of caspase- 3 revealed the caspase-dependency of the apoptotic process was. ${ }^{23}$

This is also in agreement with Chagin et al., who observed chondrocyte apoptosis on day 1 after the MIA injection. Condylar apoptosis reached a peak on day 3 , resulting in the hypocellular changes in the cartilage and disc. They found that chondrocyte apoptosis in the early stages was an essential initiator of cartilage disintegration. ${ }^{24}$

In our study, the caspase- 3 values decreased in both of the treated groups. These findings coincide with Lee et al. who reported that dexamethasone had an antiapoptotic effect on gentamicin-induced ototoxicity. ${ }^{25}$ The protective mechanism involved hindering the mitochondrial apoptosis pathway and the down-regulation of the pro-apoptotic protein Bax. ${ }^{25}$

Our results are also in agreement with Lin et al., who demonstrated that LLLT improved the cartilage structure, prevented articular cartilage disintegration and noticeably reduced the expression of caspase- 3 in a surgeryinduced OA model in rabbits. ${ }^{26}$

In our study, we used CBCT to evaluate bony changes in TMJ. Since the early 1980 s, computed tomography (CT) has been one of the most preferred modalities of imaging for assessing the osseous changes in the condyle, temporal bone, and other articulating and non-articulating surfaces in TMJ. The accuracy of CT in detecting osseous changes is as high as $87 \%$. The images were obtained in the sagittal plane, which is desirable for the assessment of osteophytes, erosion, flattening, and sclerosis. ${ }^{27}$

In our study, in the untreated osteoarthritic group, the condyle revealed erosion, flattening of the articular surface of the condylar head and osteophyte formation at 
1 week. At 4 weeks, sclerosis was observed. This concurs with a study conducted by Wang et al., in which erosion was observed at 1 and 2 weeks after OA induction. ${ }^{10}$ However, osteophytes were observed at 4 weeks and sclerosis at 8 weeks. ${ }^{10}$ This was attributed to the fact that MIA-induced arthritis progressed in a dose-dependent manner. The higher the dose, the greater and faster the destruction. In our study, OA was induced by the IA injection of $2 \mathrm{mg} /$ joint of MIA, while in the study by Wang et al., a lower dose $(0.5 \mathrm{mg} /$ joint $)$ was used. The temporal bone presented with nearly no changes compared to the condyle. This resembles the radiographical evaluation performed by Mani and Sivasubramanian, who reported that the condylar bony changes were greater than the temporal bone changes. ${ }^{28}$

In our study there was a great improvement in both the CS- and LLL-treated groups. These findings are in accordance with Ringold et al., who found that patients receiving IA CS therapy showed improvement in CT images in a follow-up analysis. ${ }^{29}$ No radiographic studies were performed to assess improvement in bony structure after LLLT. However, its effectiveness in improving mandibular movements and in reducing pain have been proven clinically in many studies. ${ }^{30}$

More animal studies are required to determine the ideal physical parameters of LLLT, such as duration, energy doses and frequency. Also, recent investigatory methods must be used to assess the therapeutic effects of LLL in treating OA with a long-term follow-up. In addition, more experiments are needed to study the combined early effect of both glucocorticoids and LLL in treating acute OA.

\section{Conclusions}

From our study, it was concluded that using the present laser parameters, LLLT was as effective as dexamethasone in its formative effects, as indicated by an increase in proteoglycan synthesis. However, LLLT was superior in its anti-apoptotic effects. An in-depth radiological evaluation is important to assess disease activity, and to help plan the treatment modality and monitor the therapeutic response.

\section{References}

1. Kim JH, Ok SM, Heo JY, et al. The clinical and radiographic features of patients with temporomandibular joint osteoarthritis: Comparison of adolescents and middle-old aged Koreans. J Oral Med Pain. 2014;39:2-9.

2. Breedveld FC. Osteoarthritis - the impact of a serious disease. Rheumatol. 2004;43(Suppl 1):i4-8.

3. Galanti C, Musumeci G, Valentino J, Giunta S, Castorina S. A role for apoptosis in temporomandibular joint disc degeneration. A contemporary review. Ital J Anat Embryol. 2013;118:151-158.

4. Cho $\mathrm{BH}$, Jung $\mathrm{YH}$. Intra- and interobserver agreement of computed tomography in assessment of the mandibular condyle. Korean J Oral Maxillofac Radiol. 2007;37:191-195.
5. Clark AR, Belvisi MG. Maps and legends: The quest for dissociated ligands of the glucocorticoid receptor. Pharmacol Ther. 2012;134:54-67.

6. Stephens MB, Beutler AI, O'Connor FG. Musculoskeletal injections: A review of the evidence. Am Fam Physician. 2008;78:971-976.

7. Mountziaris PM, Kramer PR, Mikos AG. Emerging intra-articular drug delivery systems for the temporomandibular joint. Methods. 2009;47:134-140.

8. Huang Z, Ma J, Chen J, Shen B, Pei F, Kraus VB. The effectiveness of low-level laser therapy for nonspecific chronic low back pain: A systematic review and meta-analysis. Arthritis Res Ther. 2015;17:360.

9. Herman $\mathrm{JH}$, Khosla RC. In vitro effects of $\mathrm{Nd}$ :YAG laser radiation on cartilage metabolism. J Rheumatol. 1988;15:1818-1826.

10. Wang XD, Kou XX, He DQ, et al. Progression of cartilage degradation, bone resorption and pain in rat temporomandibular joint osteoarthritis induced by injection of iodoacetate. PLOS ONE. 2012;7:e45036.

11. Connolly KM, Stecher VJ, Pruden DJ. Effect of auranofin on plasma fibronectin, $C$ reactive protein, and albumin levels in arthritic rats. Ann Rheum Dis. 1988;47:515-521.

12. El-Hakim IE, Abdel-Hamid IS, Bader A. Temporomandibular joint (TMJ) response to intra-articular dexamethasone injection following mechanical arthropathy: A histological study in rats. Int J Oral Maxillofac Surg. 2005;34:305-310.

13. Khozeimeh F, Moghareabed A, Allameh M, Baradaran S. Comparative evaluation of low-level laser and systemic steroid therapy in adjuvant-enhanced arthritis of rat temporomandibular joint: $A$ histological study. Dent Res J. 2015;12:215-223.

14. Mazzetto MO, Carrasco TG, Bidinelo EF, Pizzo RC, Mazzetto RG. Low intensity laser application in temporomandibular disorders: A phase I double-blind study. Cranio. 2007;25:186-192.

15. Steven L, Wendy U, Raymond A, et al. AVMA Guidelines for the Euthanasia of Animals: 2013 Edition. Schaumburg (IL): American Veterinary Medical Association; 2013.

16. Lampropoulou-Adamidou K, Lelovas $\mathrm{P}$, Karadimas EV, et al. Useful animal models for the research of osteoarthritis. Europ J Orthop Surg Traumatol. 2014;24:263-271.

17. Guzman RE, Evans MG, Bove S, Morenko B, Kilgore K. Mono-iodoacetate-induced histologic changes in subchondral bone and articular cartilage of rat femorotibial joints: An animal model of osteoarthritis. Toxicol Pathol. 2003;31:138-139.

18. Clark AR. MAP kinase phosphatase 1: A novel mediator of biological effects of glucocorticoids? J Endocrinol. 2003;178:5-12.

19. Karu TI, Kolyakov SF. Exact action spectra for cellular responses relevant to phototherapy. Photomed Laser Surg. 2005;23:355-361.

20. Flahiff CM, Narmoneva DA, Huebner JL, Kraus VB, Guilak F, Setton LA. Osmotic loading to determine the intrinsic material properties of guinea pig knee cartilage. J Biomech. 2002;35:1285-1290.

21. Lu YC, Evans CH, Grodzinsky AJ. Effects of short-term glucocorticoid treatment on changes in cartilage matrix degradation and chondrocyte gene expression induced by mechanical injury and inflammatory cytokines. Arthritis Res Ther. 2011;13:R142.

22. Gottlieb T, Jörgensen B, Rohde E, Müller G, Scheller EE. The influence of irradiation with low-level diode laser on the proteoglycan content in arthritically changed cartilage in rabbits. Med Laser Applicat. 2006;21:53-59.

23. Kourí-Flores JB, Abbud-Lozoya KA, Roja-Morales L. Kinetics of the ultrastructural changes in apoptotic chondrocytes from an osteoarthrosis rat model: A window of comparison to the cellular mechanism of apoptosis in human chondrocytes. Ultrastruct Pathol. 2002;26:33-40.

24. Chagin AS, Karimian E, Zaman F, Takigawa M, Chrysis D, Sävendahl L. Tamoxifen induces permanent growth arrest through selective induction of apoptosis in growth plate chondrocytes in cultured rat metatarsal bones. Bone. 2007;40:1415-1424.

25. Lee JH, Oh SH, Kim TH, Go YY, Song JJ. Anti-apoptotic effect of dexamethasone in an ototoxicity model. Biomater Res. 2017;21:4.

26. Lin HD, He CQ, Luo QL, Zhang JL, Zeng DX. The effect of low-level laser to apoptosis of chondrocyte and caspases expression, including caspase- 8 and caspase- 3 in rabbit surgery-induced model of knee osteoarthritis. Rheumatol Int. 2012;32:759-766. 
27. Westesson PL. Reliability and validity of imaging diagnosis of temporomandibular joint disorder. Adv Dent Res. 1993;7:137-151.

28. Mani FM, Sivasubramanian SS. A study of temporomandibular joint osteoarthritis using computed tomographic imaging. Biomed J. 2016;39:201-206.

29. Ringold S, Torgerson TR, Egbert MA, Wallace CA. Intraarticular corticosteroid injections of the temporomandibular joint in juvenile idiopathic arthritis. J Rheumatol. 2008;35:1157-1164.

30. Mazzetto MO, Hotta TH, Pizzo RC. Measurements of jaw movements and TMJ pain intensity in patients treated with GaAlAs laser. Braz Dent J. 2010;21:356-360. 